\title{
Experimental and Theoretical Investigations of Structural Trends for Selenium(IV) Imides and Oxides: X-ray Structure of $\mathrm{Se}_{3}(\mathrm{NAd})_{2}$
}

Tiina Maaninen, Heikki M. Tuononen, Gabriele Schatte, Reijo Suontamo, Jussi Valkonen, Risto Laitinen, and Tristram Chivers

\section{Supporting Information}

Table S1. B3PW91/6-31G* Optimized Bond Lengths $(\AA)$ and Angles $\left(^{\circ}\right)$ of Sulfonyl Amines $\mathrm{RNSO}_{2}$, and Sulfinyl and Seleninyl Amines RNEO $\left(\mathrm{E}=\mathrm{S}, \mathrm{Se} ; \mathrm{R}=\mathrm{H}, \mathrm{Me},{ }^{\mathrm{t}} \mathrm{Bu}\right.$, $\left.\mathrm{SiMe}_{3} ; \mathrm{A}=\mathrm{H}, \mathrm{C}, \mathrm{Si}\right)$

Table S2. B3PW91/6-31G* Optimized Bond Lengths $(\AA)$ and Angles $\left(^{\circ}\right)$ of Sulfur and Selenium Diimide Dimers RNE $(\mu \text {-NR })_{2}$ ENR $\left(E=S, S e ; R=H, M e,{ }^{t} B u, \operatorname{SiMe}_{3} ; A=H\right.$, $\mathrm{C}, \mathrm{Si}$ ) in all-cis-conformation (conformation a, see Scheme 1)

Table S3. B3PW91/6-31G* Optimized Bond Lengths $(\AA)$ and Angles $\left(^{\circ}\right)$ of Sulfinyl and Seleninyl Amine Dimers $\mathrm{OE}(\mu-\mathrm{NR})_{2} \mathrm{EO}\left(\mathrm{E}=\mathrm{S}, \mathrm{Se} ; \mathrm{R}=\mathrm{H}, \mathrm{Me},{ }^{\mathrm{t}} \mathrm{Bu}, \mathrm{SiMe}_{3} ; \mathrm{A}=\mathrm{H}, \mathrm{C}, \mathrm{Si}\right)$ in all-cis-conformation (conformation a, see Scheme 1)

Table S4. B3PW91/6-31G* Optimized Bond Lengths $(\AA)$ and Angles $\left(^{\circ}\right)$ of OSe $(\mu$ $\mathrm{NR})_{2} \mathrm{ENR}\left(\mathrm{E}=\mathrm{S}, \mathrm{Se} ; \mathrm{R}=\mathrm{H}, \mathrm{Me},{ }^{\mathrm{t}} \mathrm{Bu}, \mathrm{SiMe}_{3}\right)$ in all-cis-conformation (conformation $\mathbf{a}$, see Scheme 1) 
Table S5. B3PW91/6-31G* Optimized Bond Lengths $(\AA)$ and Angles $\left(^{\circ}\right)$ of Various Cycloaddition Products in all-cis-conformation (conformation a, see Scheme 1) $(\mathrm{A}=\mathrm{O}$, N)

Table S6. The MP2/cc-pVDZ//B3PW91/6-31G*, CCSD/cc-pVDZ//B3PW91/6-31G*, and CCSD(T)/cc-pVDZ//B3PW91/6-31G* Total Energies (a.u.) of $\operatorname{RNSO}_{x}(x=1,2)$ and RNSeO Including the ZPE Corrections (a.u.)

Table S7. The MP2/cc-pVDZ//B3PW91/6-31G*, CCSD/cc-pVDZ//B3PW91/6-31G*, and CCSD(T)/cc-pVDZ//B3PW91/6-31G* Total Energies (a.u.) of RNE( $\mu$-NR) ${ }_{2} \mathrm{ENR}(\mathrm{E}$ $=\mathrm{S}, \mathrm{Se}$ ) Including the ZPE Corrections (a.u.)

Table S8. The MP2/cc-pVDZ//B3PW91/6-31G*, CCSD/cc-pVDZ//B3PW91/6-31G*, and $\operatorname{CCSD}(\mathrm{T}) / \mathrm{cc}-\mathrm{pVDZ} / \mathrm{B} 3 \mathrm{PW} 91 / 6-31 \mathrm{G}^{*}$ Total Energies (a.u.) of $\mathrm{OE}(\mu-\mathrm{NR})_{2} \mathrm{EO}(\mathrm{E}=$ S, Se) Including the ZPE Corrections (a.u.)

Table s9. The MP2/cc-pVDZ//B3PW91/6-31G*, CCSD/cc-pVDZ//B3PW91/6-31G*, and $\operatorname{CCSD}(\mathrm{T}) / \mathrm{cc}-\mathrm{pVDZ} / \mathrm{B} 3 \mathrm{PW} 91 / 6-31 \mathrm{G}^{*}$ Total Energies (a.u.) of $\mathrm{OSe}\left(\mu\right.$-NR) ${ }_{2} \mathrm{ENR}(\mathrm{E}$ $=\mathrm{S}, \mathrm{Se}$ ) Including the ZPE Corrections (a.u.)

Table S10. The MP2/cc-pVDZ//B3PW91/6-31G*, CCSD/cc-pVDZ//B3PW91/6-31G*, and CCSD(T)/cc-pVDZ//B3PW91/6-31G* Total Energies (a.u.) of $\mathrm{O}_{2} \mathrm{~S}\left(\mu\right.$-NR) ${ }_{2} \mathrm{SeNR}$ and $\mathrm{RNS}(\mu-\mathrm{NR})_{2} \mathrm{SeNR}$ Including the ZPE Corrections (a.u.)

Table S11. The MP2/cc-pVTZ//B3PW91/6-31G*, CCSD/cc-pVTZ//B3PW91/6-31G*, and $\operatorname{CCSD}(\mathrm{T}) / \mathrm{cc}-\mathrm{pVTZ} / \mathrm{B} 3 \mathrm{PW} 91 / 6-31 \mathrm{G}^{*}$ Total Energies (a.u.) of $\mathrm{HNSO}_{x}(x=1,2)$, $\mathrm{HNSeO}, \mathrm{HNENH}, \mathrm{HNE}(\mu-\mathrm{NH})_{2} \mathrm{ENH}, \mathrm{OE}(\mu-\mathrm{NH})_{2} \mathrm{EO}, \mathrm{O}_{2} \mathrm{~S}(\mu-\mathrm{NH})_{2} \mathrm{SeNH}$, and $\mathrm{OSe}(\mu-$ $\mathrm{NH})_{2} \mathrm{ENH}(\mathrm{E}=\mathrm{S}, \mathrm{Se})$ Including the ZPE Corrections (a.u.) 
Table S12. BSSE Uncorrected $(\Delta \mathrm{E})$ and Corrected $\left(\triangle \mathrm{E}_{\mathrm{BSSE}}\right)$ Cyclodimerization and Cycloaddition Reaction Energies ( $\mathrm{kJ}^{\mathrm{mol}}{ }^{-1}$ ) Calculated at the $\operatorname{CCSD}(\mathrm{T}) / \mathrm{cc}-$ pVTZ//B3PW91/6-31G* Level of Theory 
Table S1. B3PW91/6-31G* Optimized Bond Lengths $(\AA)$ and Angles $\left(^{\circ}\right)$ of Sulfonyl Amines $\mathrm{RNSO}_{2}$, and Sulfinyl and Seleninyl Amines RNEO (E $=\mathrm{S}, \mathrm{Se} ; \mathrm{R}=\mathrm{H}, \mathrm{Me},{ }^{\mathrm{t}} \mathrm{Bu}$, $\left.\mathrm{SiMe}_{3} ; \mathrm{A}=\mathrm{H}, \mathrm{C}, \mathrm{Si}\right)$

\begin{tabular}{|c|c|c|c|c|c|c|}
\hline Molecule & Conformation & $r(\mathrm{EN})$ & $r(\mathbf{E O})$ & $r(\mathbf{N A})$ & $\angle \mathrm{NEO}$ & $\angle$ ENA \\
\hline \multicolumn{7}{|l|}{$\mathrm{RNSO}_{2}$} \\
\hline $\mathrm{H}$ & & 1.525 & $1.454^{d}$ & 1.023 & $119.6^{d}$ & 109.5 \\
\hline $\mathrm{Me}$ & & 1.519 & $1.456^{d}$ & 1.462 & $119.7^{d}$ & 119.6 \\
\hline${ }^{t} \mathrm{Bu}$ & & 1.517 & $1.460^{d}$ & 1.491 & $120.3^{d}$ & 123.2 \\
\hline $\mathrm{SiMe}_{3}$ & & 1.499 & $1.460^{d}$ & 1.797 & $120.8^{d}$ & 127.3 \\
\hline \multicolumn{7}{|l|}{ RNSO } \\
\hline \multirow[t]{3}{*}{$\mathrm{H}$} & anti & 1.540 & 1.469 & 1.026 & 114.3 & 107.8 \\
\hline & syn & 1.532 & 1.477 & 1.026 & 120.1 & 116.0 \\
\hline & expt1. ${ }^{a}$ & $1.512(5)$ & $1.451(5)$ & $1.029(10)$ & $120.4(5)$ & $115.8(10)$ \\
\hline \multirow[t]{3}{*}{$\mathrm{Me}$} & anti & 1.543 & 1.473 & 1.455 & 114.5 & 115.6 \\
\hline & syn & 1.535 & 1.482 & 1.450 & 119.5 & 123.5 \\
\hline & exptl. $^{b}$ & 1.51 & 1.45 & 1.47 & 121 & 122 \\
\hline \multirow[t]{2}{*}{${ }^{t} \mathrm{Bu}$} & anti & 1.539 & 1.476 & 1.472 & 113.8 & 121.0 \\
\hline & syn & 1.528 & 1.485 & 1.473 & 121.3 & 129.2 \\
\hline \multirow[t]{3}{*}{$\mathrm{SiMe}_{3}$} & anti & 1.517 & 1.474 & 1.772 & 116.2 & 131.5 \\
\hline & syn & 1.515 & 1.483 & 1.793 & 120.3 & 132.9 \\
\hline & $\operatorname{exptl}^{c}$ & $1.508(5)$ & $1.444(4)$ & $1.750(6)$ & $122.5(10)$ & $133.9(9)$ \\
\hline
\end{tabular}

$\mathrm{RNSeO}$

\begin{tabular}{lcccccc}
$\mathrm{H}$ & anti & 1.696 & 1.627 & 1.029 & 109.6 & 105.6 \\
& syn & 1.688 & 1.635 & 1.029 & 115.3 & 110.2 \\
$\mathrm{Me}$ & anti & 1.701 & 1.629 & 1.452 & 110.1 & 114.9 \\
& syn & 1.694 & 1.640 & 1.447 & 114.7 & 119.9 \\
${ }^{\mathrm{t}} \mathrm{Bu}$ & anti & 1.698 & 1.632 & 1.466 & 109.1 & 119.0 \\
& syn & 1.687 & 1.646 & 1.468 & 117.6 & 125.6 \\
$\mathrm{SiMe}_{3}$ & anti & 1.676 & 1.630 & 1.770 & 111.6 & 122.0 \\
& syn & 1.670 & 1.643 & 1.786 & 116.2 & 126.2 \\
\hline
\end{tabular}

${ }^{a}$ Ref. $46{ }^{b}$ Ref. $47 .{ }^{c}$ Ref. $45 .{ }^{d}$ Mean value. 
Table S2. B3PW91/6-31G* Optimized Bond Lengths $(\AA)$ and Angles $\left(^{\circ}\right)$ of Sulfur and Selenium Diimide Dimers RNE( $\mu$-NR $)_{2}$ ENR $\left(\mathrm{E}=\mathrm{S}, \mathrm{Se} ; \mathrm{R}=\mathrm{H}, \mathrm{Me},{ }^{\mathrm{t}} \mathrm{Bu}, \mathrm{SiMe}_{3} ; \mathrm{A}=\mathrm{H}, \mathrm{C}, \mathrm{Si}\right.$ ) in all-cis-conformation (conformation a, see Scheme 1) ${ }^{a}$

\begin{tabular}{|c|c|c|c|c|c|c|c|c|c|}
\hline \multicolumn{2}{|c|}{ Molecule } & $r\left(\mathbf{E N}_{\mathrm{c}}\right)$ & $r(\mathbf{E N})$ & $r\left(\mathbf{N}_{\mathrm{c}} \mathbf{A}\right)$ & $r(\mathrm{NA})$ & $\angle \mathbf{E N}_{\mathrm{c}} \mathbf{E}$ & $\angle \mathbf{N}_{\mathrm{c}} \mathbf{E N}_{\mathrm{c}}$ & $\angle \mathbf{N E N}_{\mathrm{c}}$ & $\tau_{\text {ENEN }}$ \\
\hline \multicolumn{10}{|c|}{$\operatorname{RNE}(\mu \text {-NR })_{2}$ ENR } \\
\hline \multirow[t]{4}{*}{$\mathrm{E}=\mathrm{S}$} & $\mathrm{H}$ & 1.768 & 1.561 & 1.023 & 1.024 & 99.2 & 79.5 & 110.3 & 12.1 \\
\hline & $\mathrm{Me}$ & 1.772 & 1.557 & 1.453 & 1.461 & 99.3 & 78.6 & 110.9 & 15.6 \\
\hline & ${ }^{t} \mathrm{Bu}$ & 1.749 & 1.557 & 1.472 & 1.482 & 102.0 & 77.2 & 112.4 & 9.7 \\
\hline & $\mathrm{SiMe}_{3}$ & 1.749 & 1.540 & 1.782 & 1.750 & 100.5 & 79.5 & 111.5 & 1.6 \\
\hline \multirow[t]{4}{*}{$\mathrm{E}=\mathrm{Se}$} & $\mathrm{H}$ & 1.921 & 1.709 & 1.031 & 1.026 & 97.3 & 82.6 & 104.3 & 2.4 \\
\hline & $\mathrm{Me}$ & 1.927 & 1.707 & 1.457 & 1.460 & 98.2 & 79.1 & 106.8 & 17.6 \\
\hline & ${ }^{\mathrm{t}} \mathrm{Bu}$ & 1.900 & 1.711 & 1.470 & 1.481 & 102.1 & 76.8 & 110.3 & 11.3 \\
\hline & $\mathrm{SiMe}_{3}$ & 1.890 & 1.690 & 1.771 & 1.755 & 100.6 & 79.3 & 108.0 & 4.2 \\
\hline
\end{tabular}

${ }^{a}$ Endocyclic nitrogen atoms are denoted by a subscript $\mathrm{c}$. 
Table S3. B3PW91/6-31G* Optimized Bond Lengths $(\AA)$ and Angles $\left(^{\circ}\right)$ of Sulfinyl and Seleninyl Amine Dimers OE $(\mu-\mathrm{NR})_{2} \mathrm{EO}(\mathrm{E}$ $=\mathrm{S}, \mathrm{Se} ; \mathrm{R}=\mathrm{H}, \mathrm{Me},{ }^{\mathrm{t}} \mathrm{Bu}, \mathrm{SiMe}_{3} ; \mathrm{A}=\mathrm{H}, \mathrm{C}, \mathrm{Si}$ ) in all-cis-conformation (conformation a, see Scheme 1)

\begin{tabular}{|c|c|c|c|c|c|c|c|c|}
\hline \multicolumn{2}{|c|}{ Molecule } & $r(\mathbf{E N})$ & $r(\mathrm{EO})$ & $r(\mathbf{N A})$ & $\angle \mathbf{E N E}$ & $\angle \mathrm{NEN}$ & $\angle \mathrm{OEN}$ & $\tau_{\text {ENEN }}$ \\
\hline \multicolumn{9}{|c|}{$\mathrm{OE}(\mu-\mathrm{NR})_{2} \mathrm{EO}$} \\
\hline \multirow[t]{4}{*}{$\mathrm{E}=\mathrm{S}$} & $\mathrm{H}$ & 1.755 & 1.476 & 1.020 & 99.4 & 78.3 & 112.4 & 16.3 \\
\hline & $\mathrm{Me}$ & 1.758 & 1.481 & 1.455 & 98.6 & 78.7 & 112.1 & 16.9 \\
\hline & ${ }^{\mathrm{t}} \mathrm{Bu}$ & 1.737 & 1.483 & 1.476 & 101.0 & 78.2 & 113.6 & 9.7 \\
\hline & $\mathrm{SiMe}_{3}$ & 1.737 & 1.481 & 1.795 & 99.3 & 80.7 & 112.3 & 2.1 \\
\hline \multirow[t]{5}{*}{$\mathrm{E}=\mathrm{Se}$} & $\mathrm{H}$ & 1.916 & 1.630 & 1.026 & 97.2 & 81.1 & 108.6 & 14.0 \\
\hline & $\mathrm{Me}$ & 1.926 & 1.640 & 1.426 & 96.8 & 80.3 & 108.3 & 18.4 \\
\hline & ${ }^{t} \mathrm{Bu}$ & 1.890 & 1.639 & 1.477 & 100.2 & 78.2 & 111.6 & 13.6 \\
\hline & $\operatorname{exptl}^{a, b}$ & 1.885 & 1.621 & 1.490 & 97.8 & 79.1 & 104.9 & 18.1 \\
\hline & $\mathrm{SiMe}_{3}$ & 1.883 & 1.637 & 1.787 & 98.7 & 81.3 & 109.3 & 0.7 \\
\hline
\end{tabular}

${ }^{a}$ Ref. 11. ${ }^{b}$ Mean values. 
Table S4. B3PW91/6-31G* Optimized Bond Lengths $(\AA)$ and Angles $\left(^{\circ}\right)$ of OSe $(\mu-\mathrm{NR})_{2} \mathrm{ENR}\left(\mathrm{E}=\mathrm{S}, \mathrm{Se} ; \mathrm{R}=\mathrm{H}, \mathrm{Me},{ }^{\mathrm{t}} \mathrm{Bu}, \mathrm{SiMe}\right)$ in all-cis-conformation (conformation a, see Scheme 1) ${ }^{a}$

\begin{tabular}{|c|c|c|c|c|c|c|c|c|c|c|}
\hline \multicolumn{2}{|c|}{ Molecule } & $r\left(\mathrm{SeN}_{\mathrm{c}}\right)$ & $r\left(\mathbf{E N}_{\mathrm{c}}\right)$ & $r(\mathbf{E N})$ & $r(\mathrm{SeO})$ & $\angle \mathrm{SeN}_{\mathrm{c}} \mathrm{E}$ & $\angle \mathbf{N}_{\mathrm{c}} \mathrm{SeN}_{\mathrm{c}}$ & $\angle \mathbf{N}_{\mathbf{c}} \mathbf{E N}_{\mathbf{c}}$ & $\angle \mathbf{N E N}_{\mathrm{c}}$ & $\tau_{\text {SeNEN }}$ \\
\hline \multicolumn{11}{|c|}{$\operatorname{OSe}(\mu \text {-NR })_{2} \mathrm{ENR}$} \\
\hline \multirow[t]{4}{*}{$\mathrm{E}=\mathrm{S}$} & $\mathrm{H}$ & 1.910 & 1.766 & 1.561 & 1.630 & 98.5 & 76.4 & 84.0 & 109.1 & 12.7 \\
\hline & $\mathrm{Me}$ & 1.905 & 1.781 & 1.557 & 1.636 & 98.2 & 76.0 & 82.4 & 109.9 & 17.2 \\
\hline & ${ }^{\mathrm{t}} \mathrm{Bu}$ & 1.874 & 1.762 & 1.557 & 1.641 & 101.4 & 74.8 & 80.4 & 112.6 & 10.8 \\
\hline & $\mathrm{SiMe}_{3}$ & 1.878 & 1.750 & 1.543 & 1.638 & 99.6 & 77.0 & 83.8 & 111.1 & 0.6 \\
\hline \multirow[t]{5}{*}{$\mathrm{E}=\mathrm{Se}$} & $\mathrm{H}$ & 1.917 & 1.923 & 1.706 & 1.632 & 97.4 & 82.3 & 82.0 & 104.8 & 7.5 \\
\hline & $\mathrm{Me}$ & 1.911 & 1.939 & 1.704 & 1.637 & 97.5 & 80.2 & 78.9 & 106.2 & 18.4 \\
\hline & ${ }^{t} \mathrm{Bu}$ & 1.916 & 1.874 & 1.706 & 1.643 & 101.2 & 78.5 & 76.4 & 110.2 & 12.5 \\
\hline & $\operatorname{exptl}^{b, c}$ & 1.937 & 1.864 & 1.687 & 1.628 & 98.9 & 80.6 & 77.0 & 113.3 & 16.2 \\
\hline & $\mathrm{SiMe}_{3}$ & 1.901 & 1.875 & 1.687 & 1.640 & 99.6 & 80.9 & 79.6 & 108.4 & 2.5 \\
\hline
\end{tabular}

${ }^{a}$ Endocyclic nitrogen atoms are denoted by a subscript c. ${ }^{b}$ Ref. $14 .{ }^{c}$ Mean values. 
Table S5. B3PW91/6-31G* Optimized Bond Lengths $(\AA)$ and Angles $\left(^{\circ}\right)$ of Various Cycloaddition Products in all-cis-conformation (conformation a, see Scheme 1) $(\mathrm{A}=\mathrm{O}, \mathrm{N})^{a}$

\begin{tabular}{|c|c|c|c|c|c|c|c|c|c|c|}
\hline Molecule & $r\left(\mathrm{SeN}_{\mathrm{c}}\right)$ & $r\left(\mathbf{S N}_{\mathrm{c}}\right)$ & $r(\mathrm{SeN})$ & $r(\mathbf{S A})$ & $\angle \mathrm{SeN}_{\mathrm{c}} \mathrm{S}$ & $\angle \mathbf{N}_{\mathrm{c}} \mathrm{SeN}_{\mathrm{c}}$ & $\angle \mathbf{N}_{\mathrm{c}} \mathbf{S N}_{\mathrm{c}}$ & $\angle \mathbf{N S e N}_{\mathrm{c}}$ & $\angle \mathbf{N S N}_{\mathrm{c}}$ & $\tau_{\mathrm{SeNSN}}$ \\
\hline \multicolumn{11}{|l|}{$\mathrm{O}_{2} \mathrm{~S}(\mu \text {-NR })_{2} \mathrm{SeNR}$} \\
\hline $\mathrm{H}^{b}$ & 1.960 & 1.720 & 1.700 & 1.452 & 96.1 & 76.8 & 88.3 & 105.8 & - & 13.0 \\
\hline $\mathrm{Me}$ & 1.940 & 1.715 & 1.699 & $1.457^{b}$ & 95.6 & 75.6 & 87.7 & 106.1 & - & 18.6 \\
\hline${ }^{\mathrm{t}} \mathrm{Bu}$ & 1.927 & 1.691 & 1.699 & $1.462^{b}$ & 98.2 & 73.8 & 86.4 & 109.4 & - & 14.5 \\
\hline exptl. ${ }^{b, c}$ & 1.948 & 1.645 & 1.666 & 1.435 & 97.1 & 73.3 & 90.0 & 113.3 & - & 8.9 \\
\hline $\mathrm{SiMe}_{3}$ & 1.917 & 1.681 & 1.679 & $1.460^{b}$ & 97.5 & 75.6 & 88.8 & 107.6 & - & 5.7 \\
\hline \multicolumn{11}{|l|}{$\operatorname{RNS}(\mu-\mathrm{NR})_{2} \operatorname{SeNR}$} \\
\hline $\mathrm{H}$ & 1.918 & 1.767 & 1.706 & 1.564 & 98.3 & 77.1 & 85.1 & 106.1 & 108.6 & 8.3 \\
\hline $\mathrm{Me}$ & 1.923 & 1.771 & 1.705 & 1.559 & 98.8 & 74.9 & 82.7 & 107.7 & 110.1 & 17.3 \\
\hline${ }^{\mathrm{t}} \mathrm{Bu}$ & 1.902 & 1.747 & 1.708 & 1.561 & 102.0 & 73.1 & 80.9 & 110.2 & 112.6 & 11.0 \\
\hline $\mathrm{SiMe}_{3}$ & 1.895 & 1.741 & 1.685 & 1.558 & 100.5 & 75.4 & 83.5 & 108.5 & 111.1 & 2.1 \\
\hline
\end{tabular}

${ }^{a}$ Endocyclic nitrogen atoms are denoted by a subscript c. ${ }^{b}$ Ref. $14 .{ }^{c}$ Mean values. 
Table S6. The MP2/cc-pVDZ//B3PW91/6-31G*, CCSD/cc-pVDZ//B3PW91/6$31 \mathrm{G}^{*}$, and $\mathrm{CCSD}(\mathrm{T}) / \mathrm{cc}-\mathrm{pVDZ} / \mathrm{B} 3 \mathrm{PW} 91 / 6-31 \mathrm{G}^{*}$ Total Energies (a.u.) of $\mathrm{RNSO}_{x}(x$ $=1,2$ ) and $\mathrm{RNSeO}$ Including the ZPE Corrections (a.u.)

\begin{tabular}{cccccc}
\hline Molecule & Conformation & MP2 & CCSD & CCSD(T) & ZPE \\
\hline $\mathrm{RNSO}_{2}$ & & & & \\
$\mathrm{H}$ & -602.83526 & -602.83464 & -602.86197 & 0.02343 \\
$\mathrm{Me}$ & -642.00681 & -642.01869 & -642.05150 & 0.05171 \\
${ }^{\mathrm{t}} \mathrm{Bu}$ & -759.56420 & -759.61069 & -759.66005 & 0.13664 \\
$\mathrm{SiMe}_{3}$ & -1010.63828 & -1010.68991 & -1010.73299 & 0.12628
\end{tabular}

RNSO

$\begin{array}{lccccc}\mathrm{H} & \text { anti } & -527.83333 & -527.83924 & -527.86112 & 0.01833 \\ & \text { syn } & -527.84211 & -527.84738 & -527.86947 & 0.01839 \\ \mathrm{Me} & \text { anti } & -567.00614 & -567.02442 & -567.05174 & 0.04682 \\ & \text { syn } & -567.01872 & -567.03619 & -567.06371 & 0.04696 \\ \mathrm{t} \mathrm{Bu} & \text { anti } & -684.56097 & -684.61377 & -684.65721 & 0.13164 \\ & \text { syn } & -684.57472 & -684.62646 & -684.67031 & 0.13222 \\ \mathrm{SiMe}_{3} & \text { anti } & -935.63343 & -935.69054 & -935.73042 & 0.12140 \\ & \text { syn } & -935.64410 & -935.70049 & -935.74080 & 0.12160\end{array}$

$\mathrm{RNSeO}$

\begin{tabular}{lccccc}
$\mathrm{H}$ & anti & -2530.07873 & -2530.07780 & -2530.10320 & 0.01689 \\
& syn & -2530.08747 & -2530.08640 & -2530.11165 & 0.01695 \\
$\mathrm{Me}$ & anti & -2569.25057 & -2569.26275 & -2569.29345 & 0.04513 \\
& syn & -2569.26333 & -2569.27489 & -2569.30572 & 0.04528 \\
$\mathrm{t} \mathrm{Bu}$ & anti & -2686.80710 & -2686.85280 & -2686.89972 & 0.13012 \\
& syn & -2686.82135 & -2686.86614 & -2686.91354 & 0.13060 \\
$\mathrm{SiMe}_{3}$ & anti & -2937.87989 & -2937.92949 & -2937.97286 & 0.11988 \\
& syn & -2937.89108 & -2937.94014 & -2937.98392 & 0.12015 \\
\hline
\end{tabular}


Table S7. The MP2/cc-pVDZ//B3PW91/6-31G*, CCSD/cc-pVDZ//B3PW91/6-31G*, and CCSD(T)/cc-pVDZ//B3PW91/6-31G* Total Energies (a.u.) of RNE $(\mu \text {-NR })_{2} \operatorname{ENR}(E=\mathrm{S}, \mathrm{Se})$ Including the ZPE Corrections (a.u.) ${ }^{a}$

\begin{tabular}{|c|c|c|c|c|c|c|c|c|c|}
\hline \multicolumn{2}{|l|}{ Molecule } & MP2 & CCSD & $\operatorname{CCSD}(T)$ & ZPE & \multicolumn{2}{|l|}{ Molecule } & MP2 & ZPE \\
\hline \multicolumn{10}{|c|}{$\mathrm{RNS}(\mu-\mathrm{NR})_{2} \mathrm{SNR}$} \\
\hline \multirow[t]{5}{*}{$\mathrm{R}=\mathrm{H}$} & $\mathbf{a}$ & -1015.91738 & -1015.94681 & -1015.98862 & 0.06291 & $\mathrm{R}={ }^{\mathrm{t}} \mathrm{Bu}$ & $\mathbf{a}$ & -1642.83653 & 0.51497 \\
\hline & $\mathbf{b}$ & -1015.90801 & -1015.93632 & -1015.97816 & 0.06314 & & $\mathbf{b}$ & -1642.83484 & 0.51514 \\
\hline & $\mathbf{c}$ & -1015.91294 & -1015.94233 & -1015.98380 & 0.06310 & & c & -1642.83696 & 0.51495 \\
\hline & d & -1015.91268 & -1015.94251 & -1015.98437 & 0.06349 & & d & -1642.83380 & 0.51508 \\
\hline & $\mathbf{e}$ & -1015.91066 & -1015.94151 & -1015.98290 & 0.06353 & & e & -1642.83219 & 0.51501 \\
\hline \multirow{5}{*}{$\mathrm{R}=\mathrm{Me}$} & $\mathbf{a}$ & -1172.61884 & -1172.69354 & -1172.75854 & 0.17597 & $\mathrm{R}=\mathrm{SiMe}_{3}$ & $\mathbf{a}$ & -2647.13261 & 0.47317 \\
\hline & $\mathbf{b}$ & -1172.60393 & -1172.67852 & -1172.74306 & 0.17591 & & $\mathbf{b}$ & - & - \\
\hline & $\mathbf{c}$ & -1172.60985 & -1172.68477 & -1172.74937 & 0.17581 & & c & -2647.13053 & 0.47352 \\
\hline & d & -1172.61378 & -1172.68875 & -1172.75407 & 0.17618 & & d & -2647.12423 & 0.47338 \\
\hline & $\mathbf{e}$ & -1172.60576 & -1172.68178 & -1172.74638 & 0.17612 & & $\mathbf{e}$ & -2647.12519 & 0.47367 \\
\hline \multicolumn{10}{|c|}{$\mathrm{RNSe}(\mu \text {-NR })_{2} \mathrm{SeNR}$} \\
\hline \multirow[t]{5}{*}{$\mathrm{R}=\mathrm{H}$} & $\mathbf{a}$ & -5020.48177 & -5020.50247 & -5020.54830 & 0.05901 & $\mathrm{R}={ }^{\mathrm{t}} \mathrm{Bu}$ & $\mathbf{a}$ & -5647.40759 & 0.51158 \\
\hline & $\mathbf{b}$ & -5020.47308 & -5020.49096 & -5020.53792 & 0.05910 & & b & -5647.40360 & 0.51109 \\
\hline & c & -5020.47770 & -5020.49779 & -5020.54376 & 0.05898 & & c & -5647.40551 & 0.51143 \\
\hline & d & -5020.47831 & -5020.49841 & -5020.54476 & 0.05952 & & d & -5647.40619 & 0.51123 \\
\hline & $\mathbf{e}$ & -5020.47710 & -5020.49888 & -5020.54452 & 0.05961 & & $\mathbf{e}$ & -5647.40418 & 0.51126 \\
\hline \multirow[t]{5}{*}{$\mathrm{R}=\mathrm{Me}$} & $\mathbf{a}$ & -5177.18084 & -5177.24325 & -5177.31345 & 0.17223 & $\mathrm{R}=\mathrm{SiMe}_{3}$ & $\mathbf{a}$ & -6651.70783 & 0.46970 \\
\hline & $\mathbf{b}$ & -5177.16500 & -5177.22792 & -5177.29771 & 0.17175 & & $\mathbf{b}$ & - & - \\
\hline & c & -5177.17310 & -5177.23527 & -5177.30562 & 0.17188 & & c & -6651.70353 & 0.47000 \\
\hline & d & -5177.17562 & -5177.23846 & -5177.30900 & 0.17235 & & d & -6651.69858 & 0.47013 \\
\hline & $\mathbf{e}$ & -5177.17024 & -5177.23414 & -5177.30454 & 0.17174 & & e & -6651.69939 & 0.46973 \\
\hline
\end{tabular}

${ }^{a}$ For the denotation of different conformations, see Scheme 1. 
Table S8. The MP2/cc-pVDZ//B3PW91/6-31G*, CCSD/cc-pVDZ//B3PW91/6-31G*, and CCSD(T)/cc-pVDZ//B3PW91/6-31G* Total Energies (a.u.) of $\mathrm{OE}(\mu \text {-NR })_{2} \mathrm{EO}(\mathrm{E}=\mathrm{S}, \mathrm{Se})$ Including the ZPE Corrections (a.u.) ${ }^{a}$

\begin{tabular}{|c|c|c|c|c|c|c|c|c|c|c|c|}
\hline \multicolumn{2}{|l|}{ Molecule } & MP2 & CCSD & $\operatorname{CCSD}(T)$ & ZPE & \multicolumn{2}{|l|}{ Molecule } & MP2 & CCSD & $\operatorname{CCSD}(T)$ & ZPE \\
\hline \multicolumn{6}{|c|}{$\mathrm{OS}(\mu-\mathrm{NR})_{2} \mathrm{SO}$} & \multicolumn{6}{|c|}{$\mathrm{OSe}(\mu-\mathrm{NR})_{2} \mathrm{SeO}$} \\
\hline \multirow[t]{5}{*}{$\mathrm{R}=\mathrm{H}$} & $\mathbf{a}$ & -1055.65653 & -1055.67374 & -1055.71331 & 0.04067 & $\mathrm{R}=\mathrm{H}$ & $\mathbf{a}$ & -5060.18007 & -5060.18998 & -5060.23295 & 0.03678 \\
\hline & b & -1055.65365 & -1055.66888 & -1055.70893 & 0.04127 & & $\mathbf{b}$ & -5060.17386 & -5060.18207 & -5060.22589 & 0.03716 \\
\hline & c & -1055.65449 & -1055.67131 & -1055.71089 & 0.04100 & & c & -5060.17651 & -5060.18653 & -5060.22937 & 0.03686 \\
\hline & d & -1055.65343 & -1055.67104 & -1055.71105 & 0.04139 & & d & -5060.17741 & -5060.18721 & -5060.23075 & 0.03738 \\
\hline & e & -1055.64929 & -1055.66799 & -1055.70722 & 0.04092 & & $\mathbf{e}$ & -5060.17382 & -5060.18582 & -5060.22818 & 0.03693 \\
\hline \multirow[t]{5}{*}{$\mathrm{R}=\mathrm{Me}$} & $\mathbf{a}$ & -1134.01613 & -1134.05713 & -1134.10819 & 0.09763 & $\mathrm{R}=\mathrm{Me}$ & $\mathbf{a}$ & -5138.53713 & -5138.56921 & -5138.62443 & 0.09386 \\
\hline & b & -1134.00726 & -1134.04724 & -1134.09822 & 0.09754 & & $\mathbf{b}$ & -5138.52505 & -5138.55675 & -5138.61172 & 0.09355 \\
\hline & c & -1134.01089 & -1134.05169 & -1134.10246 & 0.09753 & & c & -5138.53003 & -5138.56303 & -5138.61751 & 0.09336 \\
\hline & d & -1134.01167 & -1134.05282 & -1134.10425 & 0.09767 & & d & -5138.53194 & -5138.56429 & -5138.61967 & 0.09387 \\
\hline & $\mathbf{e}$ & -1134.00674 & -1134.04900 & -1134.09967 & 0.09730 & & $\mathbf{e}$ & -5138.52824 & -5138.56266 & -5138.61699 & 0.09337 \\
\hline \multirow[t]{5}{*}{$\mathrm{R}={ }^{\mathrm{t}} \mathrm{Bu}$} & $\mathbf{a}$ & -1369.12953 & -1369.23980 & -1369.32380 & 0.26739 & $\mathrm{R}={ }^{\mathrm{t}} \mathrm{Bu}$ & $\mathbf{a}$ & -5373.65502 & -5373.75578 & -5373.84391 & 0.26354 \\
\hline & b & -1369.12761 & -1369.23630 & -1369.32104 & 0.26688 & & $\mathbf{b}$ & -5373.64554 & -5373.74446 & -5373.83355 & 0.26316 \\
\hline & c & -1369.12934 & -1369.23874 & -1369.32323 & 0.26718 & & c & -5373.65071 & -5373.75101 & -5373.83957 & 0.26356 \\
\hline & d & -1369.12770 & -1369.23793 & -1369.32266 & 0.26734 & & d & -5373.65191 & -5373.75223 & -5373.84100 & 0.26333 \\
\hline & $\mathbf{e}$ & -1369.12464 & -1369.23570 & -1369.31997 & 0.26703 & & e & -5373.64853 & -5373.75043 & -5373.83853 & 0.26313 \\
\hline \multirow[t]{5}{*}{$\mathrm{R}=\mathrm{SiMe}_{3}$} & $\mathbf{a}$ & -1871.27374 & -1871.39400 & -1871.47147 & 0.24610 & $\mathrm{R}=\mathrm{SiMe}_{3}$ & $\mathbf{a}$ & -5875.80241 & -5875.91393 & -5875.99501 & 0.24244 \\
\hline & b & - & - & - & - & & $\mathbf{b}$ & - & - & - & - \\
\hline & c & - & - & - & - & & c & - & - & - & - \\
\hline & d & -1871.26185 & -1871.38347 & -1871.46046 & 0.24562 & & d & -5875.79049 & -5875.90242 & -5875.98312 & 0.24198 \\
\hline & e & -1871.26178 & -1871.38359 & -1871.46049 & 0.24575 & & e & -5875.78980 & -5875.90277 & -5875.98329 & 0.24197 \\
\hline
\end{tabular}

${ }^{a}$ For the denotation of different conformations, see Scheme 1. 
Table S9. The MP2/cc-pVDZ//B3PW91/6-31G*, CCSD/cc-pVDZ//B3PW91/6-31G*, and CCSD(T)/cc-pVDZ//B3PW91/6-31G* Total Energies (a.u.) of $\mathrm{OSe}(\mu-\mathrm{NR})_{2} \operatorname{ENR}(\mathrm{E}=\mathrm{S}, \mathrm{Se})$ Including the ZPE Corrections (a.u.) ${ }^{a}$

\begin{tabular}{|c|c|c|c|c|c|c|c|c|c|}
\hline \multicolumn{2}{|c|}{ Molecule } & MP2 & CCSD & $\operatorname{CCSD}(T)$ & ZPE & \multicolumn{2}{|l|}{ Molecule } & MP2 & ZPE \\
\hline \multicolumn{10}{|c|}{$\mathrm{OSe}(\mu \text {-NR })_{2} \mathrm{SNR}$} \\
\hline \multirow[t]{5}{*}{$\mathrm{R}=\mathrm{H}$} & $\mathbf{a}$ & -3038.04893 & -3038.06869 & -3038.11148 & 0.04980 & $\mathrm{R}={ }^{\mathrm{t}} \mathrm{Bu}$ & $\mathbf{a}$ & -3508.24582 & 0.38930 \\
\hline & $\mathbf{b}$ & -3038.04204 & -3038.06004 & -3038.10302 & 0.05029 & & $\mathbf{b}$ & -3508.24104 & 0.38889 \\
\hline & c & -3038.04564 & -3038.06512 & -3038.10743 & 0.04999 & & c & -3508.24429 & 0.38925 \\
\hline & d & -3038.04728 & -3038.06669 & -3038.10953 & 0.05057 & & d & -3508.23942 & 0.38899 \\
\hline & e & -3038.04837 & -3038.06971 & -3038.11103 & 0.05020 & & e & -3508.24079 & 0.38904 \\
\hline \multirow[t]{5}{*}{$\mathrm{R}=\mathrm{Me}$} & $\mathbf{a}$ & -3155.57860 & -3155.63174 & -3155.69190 & 0.13508 & $\mathrm{R}=\mathrm{SiMe}_{3}$ & $\mathbf{a}$ & -4261.46812 & 0.35798 \\
\hline & $\mathbf{b}$ & -3155.56612 & -3155.61908 & -3155.67889 & 0.13480 & & $\mathbf{b}$ & - & - \\
\hline & c & -3155.57238 & -3155.62535 & -3155.68536 & 0.13481 & & c & - & - \\
\hline & d & -3155.57548 & -3155.62892 & -3155.68930 & 0.13508 & & d & -4261.45970 & 0.35793 \\
\hline & $\mathbf{e}$ & -3155.57504 & -3155.62852 & -3155.68865 & 0.13466 & & $\mathbf{e}$ & - & - \\
\hline \multicolumn{10}{|c|}{$\mathrm{OSe}(\mu \text {-NR })_{2} \mathrm{SeNR}$} \\
\hline \multirow[t]{5}{*}{$\mathrm{R}=\mathrm{H}$} & $\mathbf{a}$ & -5040.31400 & -5040.33076 & -5040.37440 & 0.04791 & $\mathrm{R}={ }^{\mathrm{t}} \mathrm{Bu}$ & $\mathbf{a}$ & -5510.51438 & 0.38784 \\
\hline & $\mathbf{b}$ & -5040.30704 & -5040.32148 & -5040.36613 & 0.04827 & & b & -5510.50671 & 0.38678 \\
\hline & c & -5040.31070 & -5040.32714 & -5040.37079 & 0.04880 & & c & -5510.51171 & 0.38737 \\
\hline & d & -5040.31368 & -5040.32937 & -5040.37381 & 0.04857 & & d & -5510.50783 & 0.38743 \\
\hline & e & -5040.30975 & -5040.32766 & -5040.37110 & 0.04826 & & e & -5510.50914 & 0.38767 \\
\hline \multirow[t]{5}{*}{$\mathrm{R}=\mathrm{Me}$} & $\mathbf{a}$ & -5157.84262 & -5157.89096 & -5157.95298 & 0.13314 & $\mathrm{R}=\mathrm{SiMe}_{3}$ & $\mathbf{a}$ & -6263.72846 & 0.3562 \\
\hline & $\mathbf{b}$ & -5157.82856 & -5157.87716 & -5157.93876 & 0.13264 & & $\mathbf{b}$ & - & - \\
\hline & c & -5157.83591 & -5157.88409 & -5157.94608 & 0.13263 & & c & - & - \\
\hline & d & -5157.83897 & -5157.88761 & -5157.94980 & 0.13313 & & d & -6263.73818 & 0.35607 \\
\hline & e & -5157.83345 & -5157.88300 & -5157.94496 & 0.13292 & & e & - & - \\
\hline
\end{tabular}

${ }^{a}$ For the denotation of different conformations, see Scheme 1. 
Table S10. The MP2/cc-pVDZ//B3PW91/6-31G*, CCSD/cc-pVDZ//B3PW91/6-31G*, and CCSD(T)/cc-pVDZ//B3PW91/6-31G* Total Energies (a.u.) of $\mathrm{O}_{2} \mathrm{~S}(\mu \text {-NR })_{2} \operatorname{SeNR}$ and $\operatorname{RNS}(\mu \text {-NR })_{2} \mathrm{SeNR}\left(\mathrm{E}=\mathrm{S}\right.$, Se) Including the ZPE Corrections (a.u.) ${ }^{a}$

\begin{tabular}{|c|c|c|c|c|c|c|c|c|c|}
\hline \multicolumn{2}{|c|}{ Molecule } & MP2 & CCSD & $\operatorname{CCSD}(T)$ & ZPE & \multicolumn{2}{|l|}{ Molecule } & MP2 & ZPE \\
\hline \multicolumn{10}{|c|}{$\mathrm{O}_{2} \mathrm{~S}(\mu \text {-NR })_{2} \mathrm{SeNR}$} \\
\hline \multirow[t]{3}{*}{$\mathrm{R}=\mathrm{H}$} & $\mathbf{a}$ & -3113.07743 & -3113.09130 & -3113.13860 & 0.05539 & $\mathrm{R}={ }^{\mathrm{t}} \mathrm{Bu}$ & $\mathbf{a}$ & -3583.27762 & 0.39482 \\
\hline & $\mathbf{b}$ & -3113.07351 & -3113.08673 & -3113.13425 & 0.05566 & & $\mathbf{b}$ & -3583.27605 & 0.39481 \\
\hline & $\mathbf{c}$ & -3113.07645 & -3113.09065 & -3113.13754 & 0.05578 & & c & -3583.27806 & 0.39507 \\
\hline \multirow[t]{3}{*}{$\mathrm{R}=\mathrm{Me}$} & $\mathbf{a}$ & -3230.60771 & -3230.65495 & -3230.72008 & 0.14051 & $\mathrm{R}=\mathrm{SiMe}_{3}$ & $\mathbf{a}$ & -4336.49966 & 0.36323 \\
\hline & $\mathbf{b}$ & -3230.60065 & -3230.64815 & -3230.71297 & 0.14054 & & $\mathbf{b}$ & -4336.49362 & 0.36323 \\
\hline & c & -3230.60315 & -3230.65097 & -3230.71566 & 0.14049 & & c & -4336.49711 & 0.36338 \\
\hline \multicolumn{10}{|c|}{$\mathrm{RNS}(\mu-\mathrm{NR})_{2} \mathrm{SeNR}$} \\
\hline \multirow[t]{6}{*}{$\mathrm{R}=\mathrm{H}$} & $\mathbf{a}$ & -3018.18260 & -3018.20907 & -3018.25206 & 0.06060 & $\mathrm{R}={ }^{\mathrm{t}} \mathrm{Bu}$ & $\mathbf{a}$ & -3645.09963 & 0.51320 \\
\hline & $\mathbf{b}$ & -3018.17370 & -3018.19817 & -3018.24175 & 0.06117 & & $\mathbf{b}$ & -3645.10307 & 0.51315 \\
\hline & c & -3018.17842 & -3018.20451 & -3018.24744 & 0.06099 & & c & -3645.10402 & 0.51310 \\
\hline & d & -3018.17980 & -3018.20576 & -3018.24918 & 0.06162 & & d & -3645.09953 & 0.51334 \\
\hline & $\mathbf{e}$ & -3018.17694 & -3018.20454 & -3018.24730 & 0.06158 & & e & -3645.10105 & 0.51350 \\
\hline & $\mathbf{f}$ & -3018.17764 & -3018.20423 & -3018.24743 & 0.06135 & & f & -3645.10099 & 0.51363 \\
\hline \multirow[t]{6}{*}{$\mathrm{R}=\mathrm{Me}$} & $\mathbf{a}$ & -3174.86724 & -3174.93744 & -3175.00378 & 0.17408 & $\mathrm{R}=\mathrm{SiMe}_{3}$ & $\mathbf{a}$ & -4649.40302 & 0.47121 \\
\hline & $\mathbf{b}$ & -3174.87464 & -3174.94439 & -3175.01110 & 0.17386 & & b & -4649.39907 & 0.47141 \\
\hline & c & -3174.88255 & -3174.95242 & -3175.01925 & 0.17396 & & c & -4649.39876 & 0.47160 \\
\hline & d & -3174.87092 & -3174.94256 & -3175.00911 & 0.17428 & & d & -4649.39231 & 0.47164 \\
\hline & $\mathbf{e}$ & -3174.87807 & -3174.94810 & -3175.01527 & 0.17384 & & e & -4649.39312 & 0.47149 \\
\hline & $\mathbf{f}$ & -3174.87685 & -3174.94749 & -3175.01446 & 0.17432 & & f & -4649.39425 & 0.47165 \\
\hline
\end{tabular}

${ }^{a}$ For the denotation of different conformations, see Scheme 1. 
Table S11. The MP2/cc-pVTZ//B3PW91/6-31G*, CCSD/cc-pVTZ//B3PW91/6-31G*, and CCSD(T)/cc-pVTZ//B3PW91/6-31G* Total Energies (a.u.) of $\mathrm{HNSO}_{x}(x=1,2), \mathrm{HNSeO}, \mathrm{HNENH}, \mathrm{HNE}(\mu-\mathrm{NH})_{2} \mathrm{ENH}, \mathrm{OE}(\mu-\mathrm{NH})_{2} \mathrm{EO}, \mathrm{O}_{2} \mathrm{~S}(\mu \text {-NH })_{2} \mathrm{SeNH}$, and OSe $(\mu$ $\mathrm{NH})_{2} \mathrm{ENH}(\mathrm{E}=\mathrm{S}, \mathrm{Se})$ Including the ZPE Corrections (a.u. $)^{a}$

\begin{tabular}{|c|c|c|c|c|c|}
\hline Molecule & Conformation & MP2 & CCSD & $\operatorname{CCSD}(\mathrm{T})$ & ZPE \\
\hline HNSO & syn & -528.05832 & -528.05817 & -528.09300 & 0.01839 \\
\hline $\mathrm{HNSeO}$ & syn & -2530.35112 & -2530.34363 & -2530.38095 & 0.01695 \\
\hline $\mathrm{HNSO}_{2}$ & & -603.14907 & 603.13966 & -603.18451 & 0.02343 \\
\hline HNSNH & syn,syn & -508.16983 & -508.17539 & -508.21074 & 0.02968 \\
\hline HNSeNH & syn,syn & -2510.47071 & -2510.46918 & -2510.50648 & 0.02822 \\
\hline $\mathrm{HNS}(\mu-\mathrm{NH})_{2} \mathrm{SNH}$ & $\mathbf{a}$ & -1016.28678 & -1016.32840 & -1016.39609 & 0.06291 \\
\hline $\mathrm{HNSe}(\mu-\mathrm{NH})_{2} \mathrm{SeNH}$ & $\mathbf{a}$ & -5020.94460 & -5020.95219 & -5021.02117 & 0.05901 \\
\hline $\mathrm{HNS}(\mu-\mathrm{NH})_{2} \mathrm{SeNH}$ & $\mathbf{a}$ & -3018.62885 & -3018.64044 & -3018.70868 & 0.06060 \\
\hline $\mathrm{OS}(\mu-\mathrm{NH})_{2} \mathrm{SO}$ & $\mathbf{a}$ & -1056.10096 & -1056.10539 & -1056.17165 & 0.04067 \\
\hline $\mathrm{OSe}(\mu-\mathrm{NH})_{2} \mathrm{SeO}$ & $\mathbf{a}$ & -5060.72067 & -5060.71551 & -5060.78414 & 0.03678 \\
\hline $\mathrm{O}_{2} \mathrm{~S}(\mu-\mathrm{NH})_{2} \mathrm{SeNH}$ & $\mathbf{a}$ & -3113.64807 & -3113.64486 & -3113.72225 & 0.05539 \\
\hline $\mathrm{OSe}(\mu-\mathrm{NH})_{2} \mathrm{SNH}$ & $\mathbf{a}$ & -3038.51716 & -3038.52236 & -3038.59045 & 0.04980 \\
\hline $\mathrm{OSe}(\mu-\mathrm{NH})_{2} \mathrm{SeNH}$ & $\mathbf{a}$ & -5040.83288 & -5040.83415 & -5040.90296 & 0.04791 \\
\hline
\end{tabular}

${ }^{a}$ For the denotation of different conformations, see Scheme 1. 
Table S12. BSSE Uncorrected $(\Delta \mathrm{E})$ and Corrected $\left(\Delta \mathrm{E}_{\mathrm{BSSE}}\right)$ Cyclodimerization and Cycloaddition Reaction Energies $\left(\mathrm{kJ} \mathrm{mol}^{-1}\right)$ Calculated at the $\operatorname{CCSD}(\mathrm{T}) / \mathrm{cc}-$ pVTZ//B3PW91/6-31G* Level of Theory ${ }^{a}$

\begin{tabular}{|c|c|c|c|}
\hline Reaction & $\Delta \mathbf{E}$ & $\mathbf{E}_{\text {BSSE }}$ & $\Delta \mathbf{E}_{\mathrm{BSSE}}$ \\
\hline $2 \mathrm{HNSNH} \rightarrow \mathrm{HNS}(\mu-\mathrm{NH})_{2} \mathrm{SNH}$ & 76 & 25 & 101 \\
\hline $2 \mathrm{HNSeNH} \rightarrow \mathrm{HNSe}(\mu-\mathrm{NH})_{2} \mathrm{SeNH}$ & -15 & 24 & 9 \\
\hline $\mathrm{HNSNH}+\mathrm{HNSeNH} \rightarrow \mathrm{HNS}(\mu-\mathrm{NH})_{2} \mathrm{SeNH}$ & 30 & 25 & 55 \\
\hline $2 \mathrm{HNSO} \rightarrow \mathrm{OS}(\mu-\mathrm{NH})_{2} \mathrm{SO}$ & 48 & 24 & 72 \\
\hline $2 \mathrm{HNSeO} \rightarrow \mathrm{OSe}(\mu-\mathrm{NH})_{2} \mathrm{SeO}$ & -51 & 23 & -28 \\
\hline $\mathrm{HNSO}_{2}+\mathrm{HNSeNH} \rightarrow \mathrm{O}_{2} \mathrm{~S}(\mu-\mathrm{NH})_{2} \mathrm{SeNH}$ & -72 & 27 & -45 \\
\hline $\mathrm{HNSeO}+\mathrm{HNSNH} \rightarrow \mathrm{OSe}(\mu-\mathrm{NH})_{2} \mathrm{SNH}$ & 12 & 24 & 36 \\
\hline $\mathrm{HNSeO}+\mathrm{HNSeNH} \rightarrow \mathrm{OSe}(\mu-\mathrm{NH})_{2} \mathrm{SeNH}$ & -34 & 23 & -11 \\
\hline
\end{tabular}

${ }^{a}$ Values include unscaled zero point energy corrections. 